\title{
Confirmation and Generalization of an Alcohol-Dependence Locus on Chromosome I0q
}

\author{
Carolien I Panhuysen ${ }^{1,2}$, Henry R Kranzler ${ }^{3}$, Yi Yu', Roger D Weiss ${ }^{4,5}$, Kathleen Brady ${ }^{6}$, James Poling ${ }^{7}$, \\ Lindsay A Farrer ${ }^{1,2,8,9,10}$ and Joel Gelernter*,6,1
}

\begin{abstract}
'Department of Medicine (Genetics Program), Boston University School of Medicine, Boston, MA, USA; ${ }^{2}$ Department of Biostatistics, Boston University School of Public Health, Boston, MA, USA; ${ }^{3}$ Departments of Psychiatry and Genetics and Developmental Biology, University of Connecticut School of Medicine, Farmington, CT, USA; ${ }^{4}$ Alcohol and Drug Abuse Treatment Program, McLean Hospital, Belmont, MA, USA; ${ }^{5}$ Department of Psychiatry, University of Harvard Medical School, Boston, MA, USA; ${ }^{6}$ Department of Psychiatry and Behavioral Sciences, Medical University of South Carolina, Charleston, SC, USA; ${ }^{7}$ Division of Human Genetics, Department of Psychiatry, Yale University School of Medicine; and VA CT Healthcare Center, West Haven, CT, USA; ${ }^{8}$ Department of Neurology, Boston University School of Medicine, Boston, MA, USA; ${ }^{9}$ Department of Genetics \& Genomics, Boston University School of Medicine, Boston, MA, USA; ${ }^{10}$ Department of Epidemiology, Boston University School of Health, Boston, MA, USA; "'Departments of Neurobiology and Genetics, Yale University School of Medicine, New Haven, CT, USA
\end{abstract}

\begin{abstract}
Several genome scans on alcohol dependence (AD) and AD-related traits have been published. In this article, we present the results of a genome-wide linkage scan on AD and several related traits in 322 European-American (EA) families, and results of additional analysis in 335 African-American (AA) families that were the subject of a previous report. All families were initially ascertained for cocaine and/or opioid dependence. Non-parametric linkage analysis in the EA sample revealed suggestive linkages on chromosomes 7 (LOD $=2.1$ at $82.8 \mathrm{cM}, p=0.0009)$ and $10(\mathrm{LOD}=3.0$ at $137.7 \mathrm{cM}, p=0.000 \mathrm{I})$. The chromosome 10 linkage peak is $20 \mathrm{cM}$ distal from a genomewide significant linkage peak we observed previously in the AA sample. Parametric linkage analysis on chromosome 10 (assuming a recessive model, $80 \%$ penetrance, disease allele frequency $=0.3$ ) resulted in LOD scores of 2.7 at $136.7 \mathrm{cM}$ and 1.9 at $121.7 \mathrm{cM}$ in the $E A$ and AA samples, respectively, with a combined sample genome-wide significant LOD score of 4.1 at $131.7 \mathrm{cM}$. To reduce heterogeneity of the AD phenotype, we also assessed linkage of chromosome 10 markers with the presence of alcohol withdrawal symptoms, one of the seven components of the DSM-IV diagnosis of AD. Suggestive evidence for linkage was observed in both populations with only 5 cM separating the location of the peak LOD scores despite a loss of power due to a smaller number of families informative for this trait. Results of our study confirm a chromosome 10 risk locus for AD in two genetically distinct populations and suggest that this locus may correspond more precisely to a specific component of the disorder.
\end{abstract}

Neuropsychopharmacology (2010) 35, I325-1332; doi:10.1038/npp.20 I0.1; published online I0 February 2010

Keywords: alcohol dependence; genetic linkage; genome-wide scan; chromosome 10q

\section{INTRODUCTION}

Alcohol dependence (AD) is a common psychiatric disorder characterized by impaired control over alcohol consumption. Estimates of the lifetime and 12-month prevalence of the disorder in the US population were 12.5 and $3.8 \%$, respectively (Hasin et al., 2007). The annual costs related to problem drinking in the United States were estimated to be nearly $\$ 185$ billion in 1998 (Harwood, 2000) due to health consequences, criminal behavior, and lost productivity.

*Correspondence: Dr J Gelernter, Department of Psychiatry, Yale University School of Medicine, VA CT Healthcare System, 950 Campbell Avenue, II6A2, West Haven, CT, 065 I6, USA,

Tel: + 203932 57। I Ext 3599, Fax: + 2039373897 ,

E-mail: joel.gelernter@yale.edu

Received 16 September 2009; revised 17 December 2009; accepted 30 December 2009
Epidemiological research, including twin, adoption, and family studies, has shown that $\mathrm{AD}$ has an important genetic component. Heritability estimates in different studies vary between 40 and 64 percent (Hopfer et al., 2003; Pagan et al., 2006; Fowler et al., 2007). From the original hypothesis that a few genes directly influence the likelihood for a person to develop $\mathrm{AD}$, a more likely picture is emerging in which many relevant genes have roles, influencing a range of intermediate characteristics (also called 'endophenotypes') that subsequently influence an individual's susceptibility to $\mathrm{AD}$.

There are several published genome-wide linkage scans for $\mathrm{AD}$, which have identified numerous possible risk loci. However, only a few reported loci met genome-wide significance. Recently, Gelernter et al. (2009) reported genome-wide significant linkage for $\mathrm{AD}$ to markers near $117.2 \mathrm{cM}$ on chromosome 10q23.3-24.1 (LOD =3.32; 
$p=0.00005$; empirical genome-wide $p=0.033$ ). Because $\mathrm{AD}$ is a clinically and etiologically heterogeneous condition, we sought to improve the likelihood of identifying susceptibility genes by studying a more homogeneous sample based on genetically relevant phenotypes. For example, there are known differences in the expression of $\mathrm{AD}$ in different population groups not only due to differences in genetic makeup but also to cultural differences (Warren et al., 2007; Scott et al., 2008; Kang et al., 2009). Thus, performing statistical analyses stratifying by race is a reasonable approach (although linkage analysis, unlike association analysis, is not susceptible to false-positive results on the basis of stratification, assuming that the sample subsets have sufficient power to detect linkage).

According to the Diagnostic and Statistical Manual of Mental Disorders, 4th edition (DSM-IV; American Psychiatric Association 1994), the diagnosis of $\mathrm{AD}$ requires that an individual meet at least three of a total of seven criteria. Five of the seven criteria are behavioral symptoms that involve compulsive or excessive use of alcohol, despite negative social and physical consequences. The remaining two items have a more physiological or neuroadaptive nature: an increased tolerance for alcohol and withdrawal symptoms when alcohol intake decreases substantially or is abruptly discontinued. Individuals can, therefore, meet the diagnostic criteria for $\mathrm{AD}$ showing a wide variety of symptoms (which can be non-overlapping in different individuals) and need not show neuroadaptive changes.

The genetic contribution to $\mathrm{AD}$ may be more directly related to intermediate phenotypes (including endophenotypes) than to the DSM-IV clinical diagnostic criteria. Kuo et al. (2006) used quantitative measures of components of the $\mathrm{AD}$ diagnosis, for example, withdrawal symptoms and alcohol tolerance, and reported several suggestive linkages that were not revealed when a categorical AD diagnosis was studied. Other authors have suggested that a critical genetically influenced component of $\mathrm{AD}$ is the development of alcohol tolerance. There are several animal models supporting this hypothesis (Martin et al., 2008).

In this article, we present the results of a genome-wide linkage study of $\mathrm{AD}$ in European-American (EA) and African-American (AA) families, separately and combined, originally ascertained for cocaine and/or opioid dependence. We also report the results of separate linkage analyses of alcohol tolerance and alcohol withdrawal symptoms.

\section{MATERIALS AND METHODS}

\section{Subject Recruitment}

All subjects included in this analyses were recruited at four sites (Yale University School of Medicine, APT Foundation, New Haven, CT; University of Connecticut Health Center, Farmington, CT; McLean Hospital, Harvard Medical School, Belmont, MA; and Medical University of South Carolina, Charleston, SC) as part of cocaine and/or opioid-dependence studies described extensively elsewhere (Gelernter et al., 2005, 2007, 2006, 2009). Families were recruited if screening suggested that the proband and at least one sibling would meet DSM-IV diagnostic criteria for cocaine and/or opioid dependence. Alcohol use or dependence was
Table I Characteristics of European-American Families

\begin{tabular}{|c|c|}
\hline Families with $\geqslant 1$ AD sib (n) & 322 \\
\hline Families with $\geqslant 1$ AD sib pair $(n)$ & 95 \\
\hline Family size (avg.) & 4.29 \\
\hline Subjects $(n)$ & 1380 \\
\hline Subjects with SSADDA data & 902 \\
\hline Subjects genotyped (n) & 803 \\
\hline Male subjects (\%) & 50.4 \\
\hline Age range and mean (SD) & 18-79; 37.5 ( 10.07$)$ \\
\hline
\end{tabular}

Abbreviations: AD, alcohol dependence; SD, standard deviation.

not a criterion for study inclusion. Individuals with a primary diagnosis of a major psychotic illness (schizophrenia or schizoaffective or bipolar disorder) were excluded. When an affected sibling pair (ASP) was recruited, additional siblings and parents were recruited whenever possible regardless of affection status. Subjects gave written informed consent as approved by the institutional review board at each clinical site, and a certificate of confidentiality for the work was obtained from $\mathrm{NIH}$ (NIDA). Characteristics of the subjects included in this study are given in Table 1.

\section{Phenotype Assessment}

An electronic version of the SSADDA (Semi-Structured Assessment for Drug Dependence and Alcoholism; Pierucci-Lagha et al., 2005) was used to establish psychiatric diagnoses. Extensive quality control procedures were implemented to enhance the reliability of the phenotypic data. The inter-rater reliability of the diagnosis of $\mathrm{AD}$ using the SSADDA was very good $(\kappa=0.66)$ and the test-retest reliability was excellent $(\kappa=0.87)$ (Pierucci-Lagha et al., 2005). Subjects were classified as having AD when they met at least three of the following seven DSM-IV (American Psychiatric Association, 1994) criteria during a 12-month period: (1) a great deal of time is spent in activities to obtain alcohol, drink alcohol or recover from its effects, (2) persistent desire or unsuccessful efforts to cut down or control drinking, (3) evidence of an increase in alcohol tolerance, (4) important social, occupational or recreational activities are given up or reduced because of substance use, (5) alcohol is often consumed in larger amounts or over a longer period than was intended, (6) alcohol withdrawal symptoms and (7) the substance use is continued despite knowledge of having a persistent or recurrent physical or psychological problem that is likely to have been caused or exacerbated by drinking. The distribution of cocaine, opioid, and nicotine dependence diagnoses among subjects with and without AD is shown in Table 2. The DSM-IV AD items no. 3 (increase in tolerance) and no. 6 (withdrawal symptoms) were also analyzed as separate phenotypes. A subject was considered to have increased tolerance when the reported number of drinks needed to have a desired effect was increased by at least $50 \%$ over that required when regular drinking was initiated. A subject was considered to have withdrawal symptoms if he or she indicated drinking to avoid withdrawal symptoms on three or more occasions, or reported two or more of the following withdrawal 
Table 2 Substance Dependence Co-morbidity Among Subjects with AD

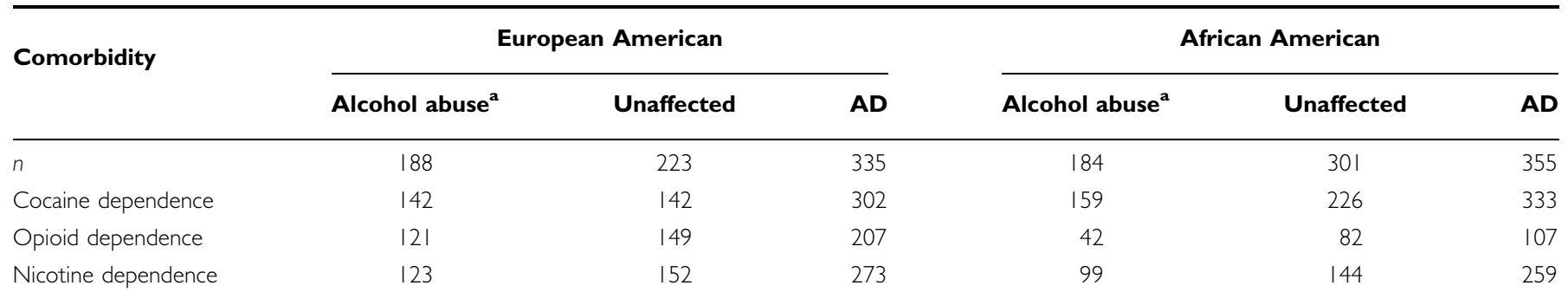

Abbreviation: AD, alcohol dependence.

a Subjects meeting diagnosis for substance abuse were excluded from the analyses.

symptoms when he or she stopped, cut down or went without drinking: (1) autonomic hyperactivity (eg, sweating or pulse rate greater than 100), (2) increased hand tremor, (3) insomnia, (4) nausea or vomiting, (5) hallucinations or illusions, (6) psychomotor agitation, (7) anxiety or (8) a grand mal seizure.

\section{Genotyping and Quality Control}

DNA was obtained from immortalized cell lines in most cases, but for a small number of subjects, DNA was obtained directly from blood or saliva. The Center for Inherited Disease Research (CIDR) genotyped 6008 single nucleotide polymorphisms (SNPs) using the Illumina Linkage IVb Marker Panel (http://www.cidr.jhmi.edu). Mega2 was used to prepare and format files for analysis (Mukhopadhyay et al., 2005). We used PedCheck (O'Connell and Weeks, 1998) and Merlin (Abecasis et al., 2002) to identify Mendelian inconsistencies. When discrepancies could not be resolved, genotypes for the marker were deleted for the entire nuclear family. We also used Merlin (Abecasis et al., 2002) to identify potentially incorrect genotypes based on estimation of the probability of double-crossover events. When a potential pedigree error was detected, ALTERTEST (McPeek and Sun, 2000) was used to determine relationships compatible with the observed genotype data. After correction, PREST (McPeek and Sun, 2000) was run to confirm that family relationship re-assignments were consistent with estimated IBD patterns. A total of 248 genotyping inconsistencies were identified out of 9570467 assays for all DNA samples from 1699 subjects (ie, $<0.003 \%$ of all assays) and these results were excluded from all subsequent analyses. Note that only certain kinds of genotyping inconsistencies are identified in this way, so this is not an overall estimate of genotyping accuracy. Three hundred forty-seven SNPs were excluded from further analysis because they were not informative in either population sample (ie, minor allele frequency less than 0.1 ). Consistency with Hardy-Weinberg equilibrium (HWE) expectations for each SNP was tested using a $\chi^{2}$-test in each ethnic group using a set of 673 unrelated subjects (one random subject from each family). Another 46 SNPs with significant evidence of deviation from HWE ( $p$-value $<0.001)$ were then excluded from further analysis. We limited our analyses to the 5633 remaining autosomal markers. The average rate of missing data among the remaining markers was $0.10 \%$.

\section{Statistical Analyses}

Assignment of subjects to populations. Differences have been identified in patterns of alcohol use and in AD risk among different populations (Hasin et al., 2007; Warren et al., 2007; Keyes et al., 2009). Also, as for any complex trait, the contribution of different putative major risk loci to the expression of AD could vary by population. It is thus important to examine the linkage findings stratified by population. For improved genetic accuracy compared with the self-reported racial background, we applied a Bayesian model-based clustering method that uses genetic data from participating subjects to infer population structure and to assign individuals probabilistically to separate populations based on the inferred cluster contributing most to an individual's ancestry (Pritchard et al., 2000). This method is implemented in the program STRUCTURE (Falush et al., 2003, 2007). We used 10000 iterations for the burn-in with a run length of 10000 . Included in the STRUCTURE analyses were 1408 SNPs from the genome-wide scan. Individuals were assigned to populations based on $>50 \%$ inferred ancestry in that population. These analyses revealed eight families whose reported ancestry differed from the population group assigned by STRUCTURE. In 10 other families, there were disagreements among family members in selfreported ancestry. These families were assigned to the population group that best fits the majority of family members based on the STRUCTURE analysis.

Linkage analysis. We assessed linkage for the DSM-IV diagnosis of $\mathrm{AD}$ and two dichotomous components of the DSM-IV diagnosis: items \# 3 ('alcohol tolerance') and \# 6 ('withdrawal symptoms'). These two items may be considered intermediate phenotypes and thus be influenced more directly by a particular gene than a complex phenotype such as AD. Linkage analyses were performed using Merlin. The entire set of markers was analyzed using a nonparametric approach for which Merlin computes the Kong and Cox allele sharing LOD score, i.e., the non-parametric equivalent of the traditional LOD score (Kong and Cox, 1997). Parametric analysis was conducted in chromosomal regions where significant evidence for linkage was obtained in the non-parametric analysis. Per our previous AD linkage 
study in a cohort of AA families (Gelernter et al., 2009), dominant and recessive models were tested assuming penetrance of $80 \%$ and a population prevalence of $7.2 \%$ for $\mathrm{AD}$ (which together correspond to allele frequencies of 0.05 for a dominant model and 0.3 for a recessive model), and allowing for linkage heterogeneity. To minimize the effects of collinearity due to inter-marker linkage disequilibrium (LD) while retaining nearly all genetic information, SNPs showing LD were grouped into clusters (Abecasis and Wigginton, 2005). These clusters included all neighboring SNPs for which the pairwise $r^{2}$ was $>0.10$. Analyses were repeated with $r^{2}$ thresholds of $0.05,0.2$, and 0.3 to evaluate the robustness of the linkage results. Map positions for the clusters were set as the midpoint between the positions of the outer-most SNPs in the cluster. In the few instances where the program encountered obligate recombinations among SNPs in a cluster, genotypes of the apparently recombinant individuals were deleted. Population haplotype frequencies were calculated using the available genotype data and a maximum-likelihood E-M algorithm. Linkage equilibrium was assumed between the clusters. Multipoint linkage analyses (parametric as well as nonparametric) were performed using SNPs and the clustered SNPs in places with LD. Parametric linkage analysis was performed using dominant and recessive models. The analyses tested for the fit of the data to a simple model with a fixed disease gene frequency $(0.05$ and 0.3 for the dominant and the recessive models, respectively) and penetrance of 0.8 . The parametric LOD scores estimated from the individual cohorts were added because they were calculated using the same parameters. To calculate HLOD scores, the program internally maximizes parameters to estimate the degree of heterogeneity in the data. The estimated HLOD scores are therefore presented separately for each cohort.

\section{RESULTS}

\section{Genomewide Linkage Analysis in the EA Population}

We performed non-parametric linkage analysis for $\mathrm{AD}$ in 95 EA families each with at least one affected sib-pair ASP. Several chromosomal locations with LOD scores exceeding 1.0 were identified; however, only two loci had LOD scores $>1.9$ (i.e., suggestive linkage) (Abecasis and Wigginton, 2005) (see Figure 1). These linkage peaks are located on chromosomes 7 (LOD score $=2.11,82.8 \mathrm{cM}$ ) and 10 (LOD score $=3.01,137.7 \mathrm{cM})$. By comparison, in our previous linkage study of $\mathrm{AD}$ in the $\mathrm{AA}$ population, we found genome-wide significant evidence (LOD score $=3.3$ ) for a locus on chromosome 10 at $117 \mathrm{cM}$ (Gelernter et al., 2009). The one LOD-unit support interval for the chromosome 10 location in the EA group is the region between $135 \mathrm{cM}$ and $146 \mathrm{cM}$.

\section{In-Depth Analysis of Chromosome 10}

To investigate further the chromosome 10 linkage findings in both populations, we performed parametric linkage analysis. Unlike non-parametric approaches, parametric linkage analysis uses information from unaffected family members in addition to the affected members and allows

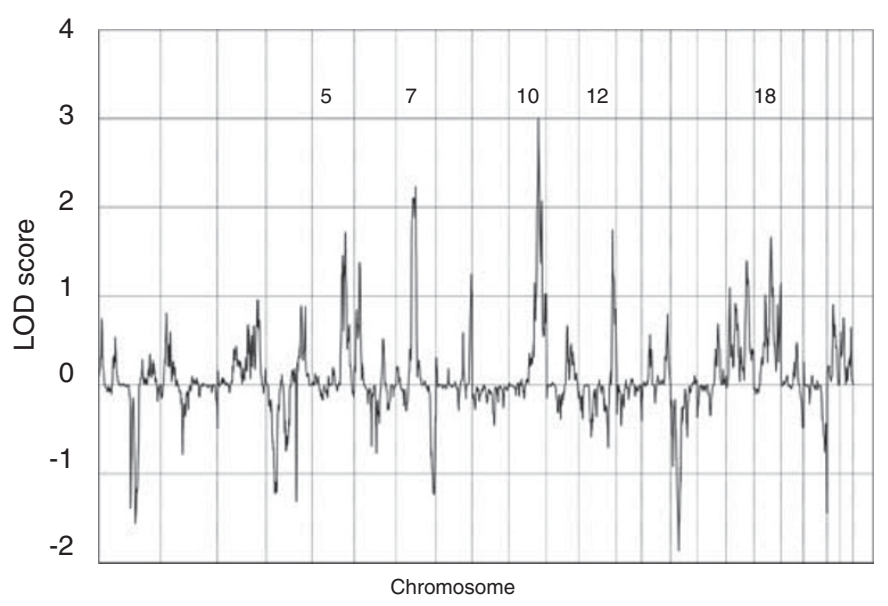

Figure I Non-parametric genome-wide linkage scan of DSM-IV diagnosis of $A D$ in 95 EA families.

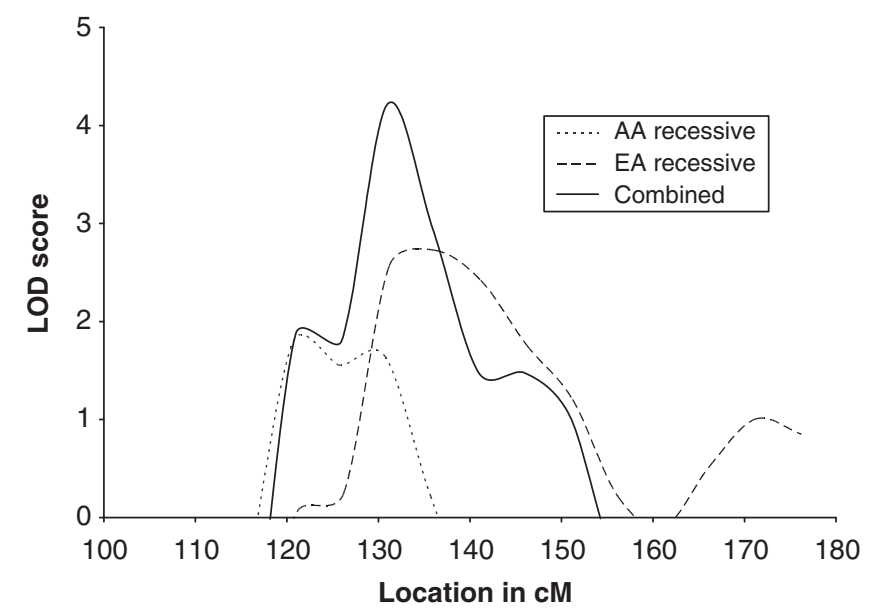

Figure 2 Cumulative parametric LOD scores of AD on chromosome 10 in EA and AA populations assuming a recessive model. (see Methods section for details). Only LOD scores greater than zero are shown.

results from analyses for each population derived separately to be added. In contrast, the procedures for pooling nonparametric analyses are much more complicated. An analysis assuming a recessive model with $80 \%$ penetrance, a disease allele frequency of 0.3 , and heterogeneity $(\alpha)$ of 0.9 yielded maximum LOD scores of 2.7 at $136.7 \mathrm{cM}$ in the EA sample and 1.9 at $121.7 \mathrm{cM}$ in the AA sample. Combining the results from both samples resulted in a maximum LOD score of 4.1 at $131.7 \mathrm{cM}$ (see Figure 2), which meets criteria for genome-wide significance (Lander and Kruglyak, 1995). Results assuming a dominant model were not as impressive: $\mathrm{LOD}=1.8$ at $136 \mathrm{cM}$ in the EA families, $\mathrm{LOD}=1.5$ at $121 \mathrm{cM}$ in the AA families, and $\mathrm{LOD}=2.6$ at $131 \mathrm{cM}$ in the total group of families.

\section{Comparison of the AD Phenotype in EAs and AAs}

To evaluate the validity of combining data from two genetically different populations, we compared the phenotypes of both groups. Table 3 shows that, although the proportion of $\mathrm{AD}$ subjects was the same in the $\mathrm{EA}$ and $\mathrm{AA}$ 
groups, severe $\mathrm{AD}$ was more prevalent in the EA group. Analysis of the individual items that contribute to the $\mathrm{AD}$ diagnosis showed that EA subjects more frequently reported increased tolerance to alcohol, impairment in social activities, withdrawal symptoms, and consumption of alcohol despite physical or psychological consequences (data not shown). Plotting the number of subjects reporting the seven DSM-IV AD criteria revealed that the individual items show a wide range of sensitivity and specificity in detecting $\mathrm{AD}$. Among subjects with two or fewer symptoms (ie, not meeting full criteria for $\mathrm{AD}$ ), only $0.5 \%$ reported withdrawal symptoms (indicating a high level of specificity); on the other hand, alcohol tolerance was reported by $37 \%$ of subjects not meeting the criteria for an $\mathrm{AD}$ diagnosis (Figure 3).

\section{Chromosome 10 Linkage Analysis of Single SSADDA items for Alcohol Withdrawal Symptoms and Increased Tolerance}

We performed linkage analysis on the SSADDA items indicating presence or absence of alcohol withdrawal and alcohol tolerance in the two population groups. There were

Table 3 Severity of AD in the European-American and AfricanAmerican Subjects

\begin{tabular}{lccccc} 
Affection & $\begin{array}{c}\text { Number of } \\
\text { positive DSM-IV } \\
\text { AD items }\end{array}$ & EA & AA & z-score & p-value \\
\hline & $n$ & 746 & 837 & & \\
Not AD & $0-2$ & $51.1 \%$ & $51.7 \%$ & 0.26 & 0.793 \\
Moderate AD & $3-5$ & $24.4 \%$ & $30.5 \%$ & 2.7 & 0.007 \\
Severe AD & $6-7$ & $24.5 \%$ & $17.8 \%$ & 3.28 & 0.001 \\
\hline
\end{tabular}

Abbreviations: AD, alcohol dependence; DSM-IV, Diagnostic and Statistical Manual of Mental Disorders, 4th Edition.
67 families (21 AA and 46 EA) with an ASP for withdrawal symptoms and 203 families (77 AA and 126 EA) with an ASP for alcohol tolerance. Non-parametric analysis of withdrawal symptoms revealed suggestive evidence for linkage in both populations: maximum LOD score $=2.0$ at $136 \mathrm{cM}$ in the AA cohort and maximum LOD score $=1.9$ at $154 \mathrm{cM}$ in the EA cohort. A secondary linkage peak at $141 \mathrm{cM}(\mathrm{LOD}=1.4)$ was observed in the EA group. These peaks are slightly more distal but much closer together than the linkage peaks for $\mathrm{AD}$ (see dashed lines in Figure 4). Results of parametric analysis were less remarkable. The most significant results were obtained for the recessive model as described above at approximately the same location: maximum LOD score $=1.5$ at $142 \mathrm{cM}$ in the EA families and maximum LOD score $=0.3$ at $145 \mathrm{cM}$ in the EA families. None of the results in either population sample were remarkable for the trait of alcohol tolerance. The highest LOD scores were 0.6 at $139.2 \mathrm{cM}$ in the EA families and 1.2 at $135.7 \mathrm{cM}$ in the AA families.

\section{DISCUSSION}

We performed a genome-wide high-density SNP linkage scan of the diagnosis of $\mathrm{AD}$ in a sample of EA families ascertained through sibling pairs affected with cocaine and/ or opioid dependence. The most significant evidence was obtained on chromosome $10 \mathrm{q}$ at $137.7 \mathrm{cM}(\mathrm{LOD}=3.0)$. This finding likely represents an independent replication of linkage observed in a similarly ascertained sample of AA families (Gelernter et al., 2009). Although such a conclusion can be disputed on the basis of the distance between the linkage peaks observed in the two populations, those peaks having their origin in the same risk locus seems to us to be the most parsimonious explanation of the data. When combining results from the EA and the AA families, the LOD score reached 4.1 at $131.7 \mathrm{cM}$. Suggestive evidence for linkage was obtained with a second locus on chromosome 7

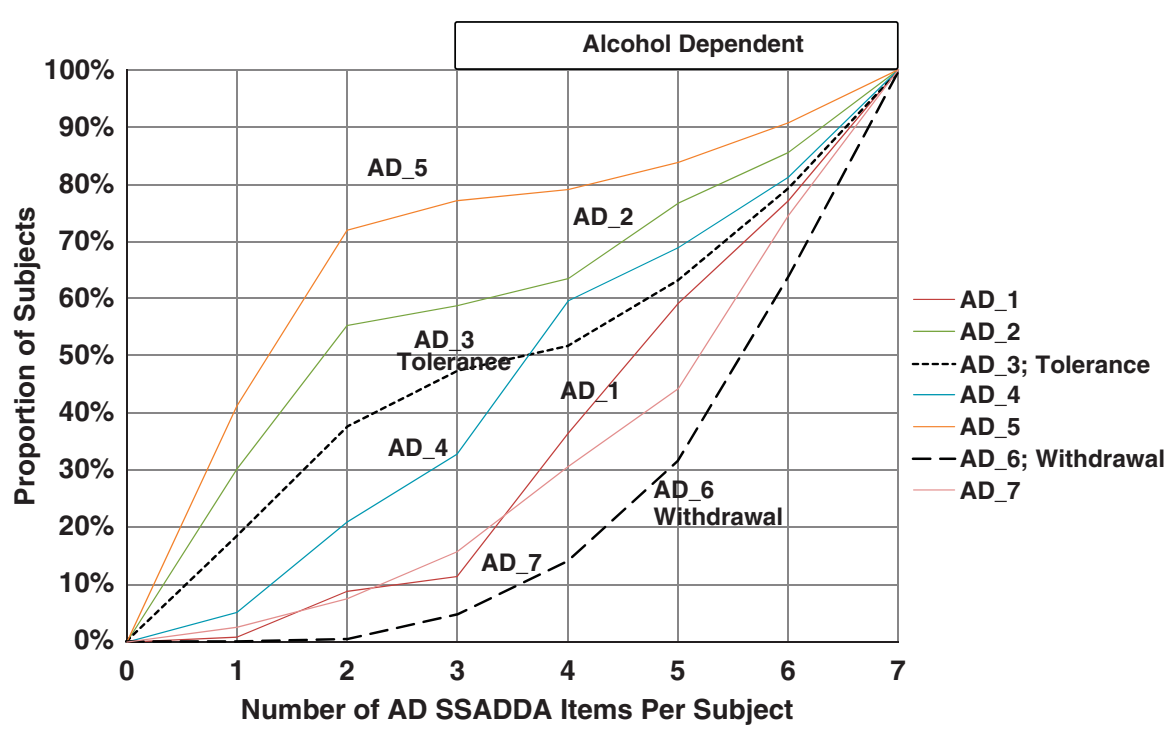

Figure 3 Cumulative percentage of subjects reporting the seven separate SSADDA items related to AD. Subjects reporting three or more items are assigned a DSM-IV diagnosis of AD. The dotted line represents the number of subjects reporting an increase in alcohol tolerance. The dashed line represents the number of subjects reporting withdrawal symptoms. 


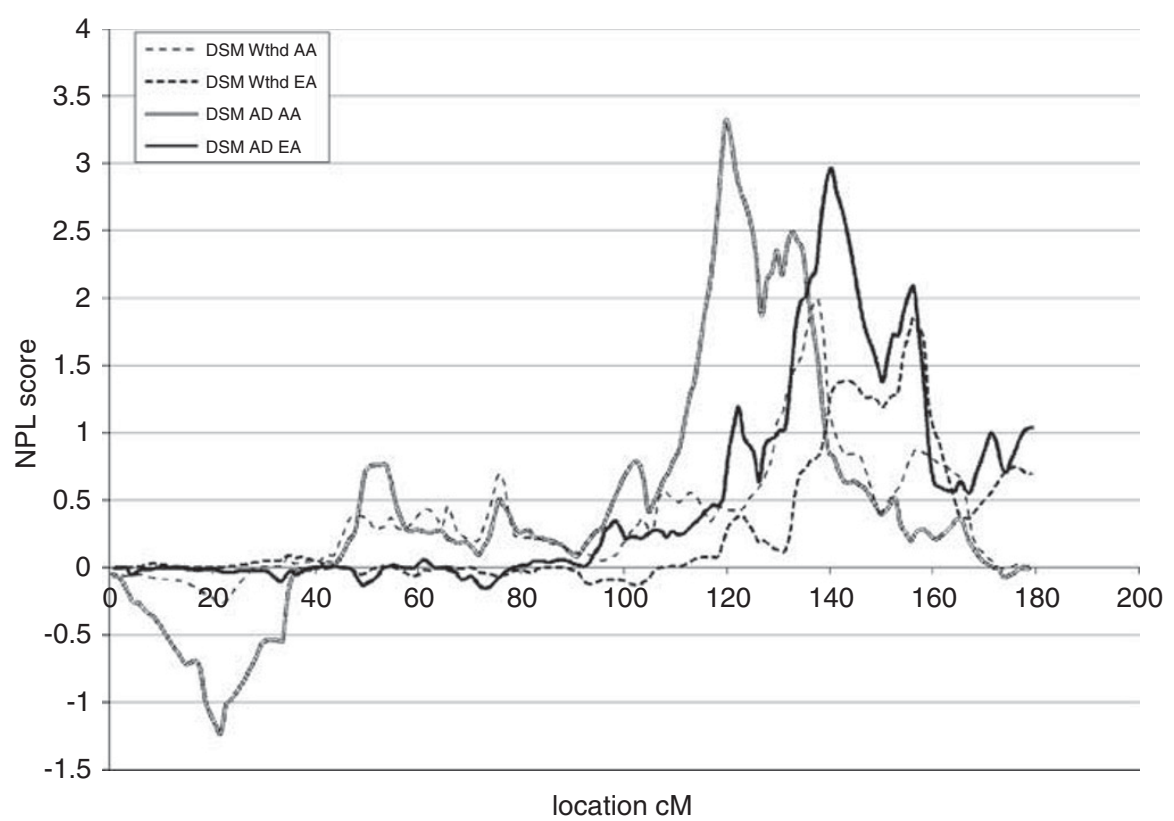

Figure 4 Non-parametric linkage results of $A D$ and alcohol withdrawal symptoms with markers on chromosome 10 in EA and AA families.

at $83 \mathrm{cM}(\mathrm{LOD}=2.1)$. Unlike the locus on chromosome 10 , this locus was not significant in the AA sample (maximum LOD score $=0.19,67 \mathrm{cM})$ (Gelernter et al., 2009).

A major concern in relation to genome-wide linkage scans is the general lack of independent replication of the results. Viewed in this light, the evidence presented here for an $\mathrm{AD}$ susceptibility locus on chromosome 10 is reassuring. Suggestive linkage evidence for this region has been reported in several other differently ascertained data sets using a variety of alcohol-dependence traits, but mostly in EA samples. The Collaborative Study on the Genetics of Alcoholism (COGA) released a set of informative families (1,350 white, non-Hispanic individuals in 143 pedigrees) for the Genetic Analysis Workshop 14 (GAW14). Zhang et al. (2005) found two suggestive linkage loci for a quantitative AD trait on chromosome 7 (96-106 cM) and on chromosome 10 (149-176 cM). Bautista et al. (2005) analyzed the same data set and reported evidence for linkage of a binary $\mathrm{AD}$ trait on chromosome $10 \mathrm{q}$ (multipoint $\mathrm{LOD}=3.68$ at $116.3 \mathrm{cM}, p=2.0 \times 10^{-5}$ ). Agrawal et al. (2008) obtained an LOD score of 2.6 for a locus on chromosome 10 at $116 \mathrm{cM}$ linked to a quantitative trait comprising a combination of alcohol and/or drug-dependence symptoms (but not to DSM-IV AD) in large genetically informative pedigrees from the COGA sample. Schuckit et al. (2005) analyzed a sample of 238 mostly EA/Hispanic sib-pairs who received an alcohol challenge test to estimate their personal response to alcohol. They reported suggestive evidence for linkage (LOD score of 2.6) with a measure of low response to alcohol to chromosome 10 at $122 \mathrm{cM}$. Viel et al. (2008) performed genome-wide linkage analysis of the rate of alcohol and cigarette consumption in an unselected Mexican-American population recruited through The San Antonio Family Heart Study. They reported a suggestive linkage peak for alcohol and smoking phenotypes near $148 \mathrm{cM}$ on chromosome 10. Bivariate (smoking and alcohol consumption) linkage analysis showed a maximum LOD score of 3.82 (genome-wide $P=0.0054$ ) at $151 \mathrm{cM}$ on chromosome 10. Similarly, Loukola et al. (2008) reported suggestive linkage for combined drinking and smoking to a locus on 10q25 in a study sample from the Finnish Twin Cohort. Thus, there is wide support for an AD-related risk locus on chromosome 10 .

The families included in this study were ascertained for DSM-IV cocaine and/or opioid dependence and have been previously evaluated for linkage to other substancedependence phenotypes (Gelernter et al., 2005, 2007, 2006, 2009). One of these traits, cocaine dependence, showed suggestive evidence of linkage to chromosome 10 (Gelernter et al., 2005), however, this locus is clearly distinct because it is nearly $50 \mathrm{cM}$ from the linkage peak for $\mathrm{AD}$ in this study. It is also unlikely that the locus on chromosome 10 is for generalized substance dependence because, in this study sample, the correlations for comorbid dependence on both alcohol and other drugs are modest (Yu et al., 2008).

In the absence of other information, the non-parametric analyses showing linkage peaks for $\mathrm{AD}$ in the $\mathrm{EA}$ and $\mathrm{AA}$ samples that are approximately $20 \mathrm{cM}$ apart support the possibility that distinct loci in this region influence $A D$ in these populations. It is plausible that multiple syntenic loci for $\mathrm{AD}$ exist in EAs and AAs in light of evidence showing population differences in clinical course, genetic variations of alcohol-metabolizing enzymes (Gelernter et al., 2009; Scott et al., 2008; Kang et al., 2009; Scott and Taylor, 2007), and known genetic differences between the populations. Moreover, we found a significantly higher proportion of severe $\mathrm{AD}$ (ie, meeting 6 or 7 DSM-IV criteria) in the EA compared with the AA group (see Table 3). However, we obtained a genome wide significant LOD score (4.1) at one chromosome 10 location in the combined samples using parametric methods that take into account linkage information from non-alcoholic relatives. The hypothesis of a single chromosome 10 locus in both populations is supported by our analyses of alcohol withdrawal symptoms showing 
linkage peaks in the two groups that are approximately $5 \mathrm{cM}$ apart (Figure 4), although the evidence for linkage is much less than for $\mathrm{AD}$, presumably because of a reduction in the number of families informative for the parametric analyses for this trait. Nonetheless, linkage analysis is too imprecise to prove or disprove the existence of multiple-linked ADrelated loci in this region. This question can be resolved more effectively by association studies in appropriately powered samples.

Although the DSM-IV diagnostic criteria provide an efficient tool to identify subjects with AD who could benefit from medical intervention, our results (consistent with numerous results from other research groups) suggest that the DSM-IV diagnosis might not be the optimal phenotype for gene discovery. More narrowly defined phenotypes may show greater correspondence with particular genes, as we observed for withdrawal symptoms. However, as our analysis showed, use of such intermediate phenotypes in linkage analyses should consider the reduction in power resulting from the smaller number of families that can be included.

The chromosomal region linked to $\mathrm{AD}$ harbors many genes, of which only a few are compelling candidate genes for the disorder. Serotonin receptor 7 (genetic locus HTR7 located at $113 \mathrm{cM}$ ) is thought to have a role in various cognitive and behavioral functions (Leo et al., 2009; Wei et al., 2009), and has been associated with schizophrenia (Erdmann et al., 1996; Ikeda et al., 2006). SLC18A2 is a vesicular amine transporter gene located at $136.4 \mathrm{cM}$; the protein encoded by this gene aids in the collection of cytosolic monoamines into synaptic vesicles across the synaptic vesicular membrane. The proper functioning of this transporter was shown to be essential for the activity of the monoaminergic systems that have been implicated in several neuropsychiatric disorders (Peter et al., 1993). Lin et al., 2005) reported an association of SLC18A2 promoter haplotypes and risk of AD.

Our findings should be considered in light of several limitations. Subjects in this study are members of families ascertained for linkage studies of cocaine and/or opioid dependence, and thus the results might not generalize to a general $\mathrm{AD}$ population. In this regard, the rate of alcohol dependence in these families is higher than the lifetime prevalence estimate of $12.5 \%$ reported for the general population (Hasin et al., 2007). Second, despite the large number of families in our sample, power to detect linkage was reduced by the availability of few parents. However, as we discussed previously (Gelernter et al., 2009), the use of a high density SNP linkage mapping panel likely compensated to some extent for the sub-optimal family structures. The loss of power was more acute in the sub-analyses of the individual SSADDA items because relatively few families had multiple sibs reporting withdrawal symptoms or increased tolerance to alcohol. To lessen this concern, we restricted these analyses to the region showing linkage with $\mathrm{AD}$.

In summary, the results of our genome-wide linkage scan indicate that a locus in the $10 \mathrm{q} 23-\mathrm{q} 24$ region contributes to the susceptibility to AD in both EAs and AAs. Our future efforts will target this region using high-density association analyses. Continued effort to understand the genetic basis of alcohol dependence is warranted by the convergence of findings reported for this chromosomal region, replicated association findings for a number of candidate genes on other chromosomes (Gelernter and Kranzler, 2009) and the considerable morbidity and mortality associated with excessive alcohol consumption.

\section{ACKNOWLEDGEMENTS}

We thank John Farrell and Michael Jervis for database management support. This work was supported by NIDA grants R01 DA12690, R01 DA12849, R01 AA11330, K24 DA15105, and K24 DA022288 and NIAAA grant K24 AA013736. Genotyping services were provided by the Center for Inherited Disease Research (CIDR). CIDR is fully funded through a federal contract from the National Institutes of Health to The Johns Hopkins University contract number N01-HG-65403.

\section{DISCLOSURE}

Dr Kranzler has received consulting fees from Ortho-McNeil Pharmaceuticals, H. Lundbeck A/S, Forest Pharmaceuticals, elbion NV, Sanofi-Aventis, Solvay Pharmaceuticals, and Alkermes, Inc. $\mathrm{He}$ has received research support from Ortho-McNeil Pharmaceuticals, Bristol-Myers Squibb Company, and Merck \& Co., Inc. and honoraria from Forest Pharmaceuticals, Alkermes, Inc., and the American College of Neuropsychopharmacology. Dr Farrer has received consulting fees from Novartis Pharmaceuticals and research support from Eisai Pharmaceuticals. Dr Gelernter reports that he has received compensation for professional services in the previous 3 years from the following entities: Yale University School of Medicine, Veterans Affairs Healthcare System (VA) and the National Institutes of Health (NIAAA, NIDA, and NIMH) and related to academic lectures and editorial functions in various scientific venues (including the ACNP). None of the other authors has anything to disclose.

\section{REFERENCES}

Abecasis GR, Cherny SS, Cookson WO, Cardon LR (2002). Merlin - rapid analysis of dense genetic maps using sparse gene flow trees. Nat Genet 30: 97-101.

Abecasis GR, Wigginton JE (2005). Handling marker-marker linkage disequilibrium: pedigree analysis with clustered markers. Am J Hum Genet 77: 754-767.

Agrawal A, Hinrichs AL, Dunn G, Bertelsen S, Dick DM, Saccone SF et al. (2008). Linkage scan for quantitative traits identifies new regions of interest for substance dependence in the Collaborative Study on the Genetics of Alcoholism (COGA) sample. Drug Alcohol Depend 93: 12-20.

American Psychiatric Association (1994). Diagnostic and Statistical Manual of Mental Disorders, 4th edn. American Psychiatric Association: Washington, DC.

Bautista JF, Quade SR, Parrado AR, Goddard KA (2005). Linkage analysis of alcoholism-related electrophysiological phenotypes: genome scans with microsatellites compared to single-nucleotide polymorphisms. BMC Genet 6(Suppl 1): S156.

Erdmann J, Nothen MM, Shimron-Abarbanell D, Rietschel M, Albus M, Borrmann M et al. (1996). The human serotonin 7 (5-HT7) receptor gene: genomic organization and systematic mutation screening in schizophrenia and bipolar affective disorder. Mol Psychiatry 1: 392-397. 
Falush D, Stephens M, Pritchard JK (2003). Inference of population structure using multilocus genotype data: linked loci and correlated allele frequencies. Genetics 164: 1567-1587.

Falush D, Stephens M, Pritchard JK (2007). Inference of population structure using multilocus genotype data: dominant markers and null alleles. Mol Ecol Notes 7: 574-578.

Fowler T, Shelton K, Lifford K, Rice F, McBride A, Nikolov I et al. (2007). Genetic and environmental influences on the relationship between peer alcohol use and own alcohol use in adolescents. Addiction 102: 894-903.

Gelernter J, Kranzler HR, Panhuysen C, Weiss RD, Brady K, Poling J et al. (2009). Dense genomewide linkage scan for alcohol dependence in African Americans: significant linkage on chromosome 10. Biol Psychiatry 65: 111-115.

Gelernter J, Kranzler HR (2009). Genetics of alcohol dependence. Hum Genet 126: 91-99.

Gelernter J, Panhuysen C, Weiss R, Brady K, Hesselbrock V, Rounsaville B et al. (2005). Genomewide linkage scan for cocaine dependence and related traits: significant linkages for a cocainerelated trait and cocaine-induced paranoia. Am J Med Genet B Neuropsychiatr Genet 136B: 45-52.

Gelernter J, Panhuysen C, Weiss R, Brady K, Poling J, Krauthammer $\mathrm{M}$ et al. (2007). Genomewide linkage scan for nicotine dependence: identification of a chromosome 5 risk locus. Biol Psychiatry 61: 119-126.

Gelernter J, Panhuysen C, Wilcox M, Hesselbrock V, Rounsaville B, Poling J et al. (2006). Genomewide linkage scan for opioid dependence and related traits. Am J Hum Genet 78: 759-769.

Harwood H (2000). Alcohol Abuse and Alcoholism. National Institutes of Health, Rockville, MD.

Hasin DS, Stinson FS, Ogburn E, Grant BF (2007). Prevalence, correlates, disability, and comorbidity of DSM-IV alcohol abuse and dependence in the United States: results from the national epidemiologic survey on alcohol and related conditions. Arch Gen Psychiatry 64: 830-842.

Hopfer CJ, Crowley TJ, Hewitt JK (2003). Review of twin and adoption studies of adolescent substance use. J Am Acad Child Adolesc Psychiatry 42: 710-719.

Ikeda $\mathrm{M}$, Iwata $\mathrm{N}$, Kitajima $\mathrm{T}$, Suzuki $\mathrm{T}$, Yamanouchi $\mathrm{Y}$, Kinoshita Y et al. (2006). Positive association of the serotonin 5-HT7 receptor gene with schizophrenia in a Japanese population. Neuropsychopharmacology 31: 866-871.

Kang TS, Woo SW, Park HJ, Lee Y, Roh J (2009). Comparison of genetic polymorphisms of CYP2E1, ADH2, and ALDH2 genes involved in alcohol metabolism in Koreans and four other ethnic groups. J Clin Pharm Ther 34: 225-230.

Keyes KM, Geier T, Grant BF, Hasin DS (2009). Influence of a drinking quantity and frequency measure on the prevalence and demographic correlates of DSM-IV alcohol dependence. Alcohol Clin Exp Res 33: 761-771.

Kong A, Cox N (1997). Allele-sharing models: LOD scores and accurate linkage tests. Am J Hum Genet 61: 1179-1188.

Kuo PH, Neale MC, Riley BP, Webb BT, Sullivan PF, Vittum J et al. (2006). Identification of susceptibility loci for alcohol-related traits in the Irish Affected Sib Pair Study of Alcohol Dependence. Alcohol Clin Exp Res 30: 1807-1816.

Lander E, Kruglyak L (1995). Genetic dissection of complex traits: guidelines for interpreting and reporting linkage results. Nat Genet 11: 241-247.

Leo D, Adriani W, Cavaliere C, Cirillo G, Marco EM, Romano E et al. (2009). Methylphenidate to adolescent rats drives enduring changes of accumbal Htr7 expression: implications for impulsive behavior and neuronal morphology. Genes Brain Behav 8: 356-368.
Lin Z, Walther D, Yu XY, Li S, Drgon T, Uhl GR (2005). SLC18A2 promoter haplotypes and identification of a novel protective factor against alcoholism. Hum Mol Genet 14: 1393-1404.

Loukola A, Broms U, Maunu H, Widen E, Heikkila K, Siivola M et al. (2008). Linkage of nicotine dependence and smoking behavior on $10 \mathrm{q}, 7 \mathrm{q}$ and $11 \mathrm{p}$ in twins with homogeneous genetic background. Pharmacogenomics J 8: 209-219.

Martin GE, Hendrickson LM, Penta KL, Friesen RM, Pietrzykowski AZ, Tapper AR et al. (2008). Identification of a BK channel auxiliary protein controlling molecular and behavioral tolerance to alcohol. Proc Natl Acad Sci USA 105: 17543-17548.

Mukhopadhyay N, Almasy L, Schroeder M, Mulvihill WP, Weeks DE (2005). Mega2: data-handling for facilitating genetic linkage and association analyses. Bioinformatics 21: 2556-2557.

McPeek MS, Sun L (2000). Statistical tests for detection of misspecified relationships by use of genome-screen data. Am J Hum Genet 66: 1076-1094.

O'Connell JR, Weeks DE (1998). PedCheck: a program for identification of genotype incompatibilities in linkage analysis. Am J Hum Genet 63: 259-266.

Pagan JL, Rose RJ, Viken RJ, Pulkkinen L, Kaprio J, Dick DM (2006). Genetic and environmental influences on stages of alcohol use across adolescence and into young adulthood. Behav Genet 36: 483-497.

Peter D, Finn JP, Klisak I, Liu Y, Kojis T, Heinzmann C et al. (1993). Chromosomal localization of the human vesicular amine transporter genes. Genomics 18: 720-723.

Pierucci-Lagha A, Gelernter J, Feinn R, Cubells JF, Pearson D, Pollastri A et al. (2005). Diagnostic reliability of the Semistructured Assessment for Drug Dependence and Alcoholism (SSADDA). Drug and Alcohol Dependence 80: 303-312.

Pritchard JK, Stephens M, Donnelly P (2000). Inference of population structure using multilocus genotype data. Genetics 155: 945-959.

Scott DM, Taylor RE (2007). Health-related effects of genetic variations of alcohol-metabolizing enzymes in African Americans. Alcohol Res Health 30: 18-21.

Scott DM, Williams CD, Cain GE, Kwagyan J, Kalu N, Ehlers CL et al. (2008). Clinical course of alcohol dependence in African Americans. J Addict Dis 27: 43-50.

Schuckit MA, Wilhelmsen K, Smith TL, Feiler HS, Lind P, Lange LA et al. (2005). Autosomal linkage analysis for the level of response to alcohol. Alcohol Clin Exp Res 29: 1976-1982.

Viel K, Charlesworth J, Tejero E, Dyer T, Cole S, Haack K et al. (2008). A linkage analysis of cigarette and alcohol consumption in an unselected Mexican American population. Am J Med Genet B Neuropsychiatr Genet 147B: 983-986.

Warren JI, Stein JA, Grella CE (2007). Role of social support and self-efficacy in treatment outcomes among clients with cooccurring disorders. Drug Alcohol Depend 89: 267-274.

Wei Z, Wang L, Xuan J, Che R, Du J, Qin S et al. (2009). Association analysis of serotonin receptor 7 gene (HTR7) and risperidone response in Chinese schizophrenia patients. Prog Neuropsychopharmacol Biol Psychiatry 33: 547-551.

Yu Y, Kranzler HR, Panhuysen C, Weiss RD, Poling J, Farrer LA et al. (2008). Substance dependence low-density whole genome association study in two distinct American populations. Hum Genet 123: 495-506.

Zhang C, Cawley S, Liu G, Cao M, Gorrell H, Kennedy GC (2005). A genomewide linkage analysis of alcoholism on microsatellite and single-nucleotide polymorphism data, using alcohol dependence phenotypes and electroencephalogram measures. BMC Genet 6(Suppl 1): S17. 